\title{
Amplification of magnetic field twisting by a stagnation point flow
}

\author{
Chloe Sumner and Youra Taroyan
}

\author{
Department of Physics, University of Aberystwyth, Aberystwyth SY23 3BZ, UK \\ e-mail: chs73@aber.ac.uk
}

Received 26 June 2020 / Accepted 10 September 2020

\begin{abstract}
Context. Flows are a common feature of many processes occurring in the solar atmosphere, such as the formation of prominences where evaporated plasma from the chromosphere condensates along thin prominence threads that are seen to twist and oscillate.

Aim. We aim to investigate the twisting of these threads by plasma condensation during their formation.

Methods. We introduce a simple model with fixed critical points where the flow speed matches the Alfvén speed. This allows us to study the problem separately in the sub-Alfvénic and super-Alfvénic regimes. The temporal and spatial evolution of small amplitude initial twists along a thread is investigated analytically and numerically.

Results. Analytical solutions are constructed in terms of the generalised hypergeometric functions. The solutions grow in time, despite the absence of any influxes of energy or magnetic fields. These results are confirmed numerically: We find oscillations with an amplifying amplitude and increasing period in the sub-Alfvénic regime. In the super-Alfvénic regime, we find twist amplification without any accompanying oscillations. An interesting result is the convergence of the twists at the critical points that leads to the formation of steep gradients and small scales. Energy is transferred from the flow to the amplifying twists.

Conclusions. Magnetic field lines may be twisted by a stagnation point flow without the influx of any azimuthal field or energy. This twisting could assist in the formation of topology that is able to support the growth of prominences. The formation of steep gradients and small scales at the critical point is a new phenomenon which requires further investigation in the non-linear regime with the inclusion of magnetic diffusion.
\end{abstract}

Key words. Sun: atmosphere - Sun: filaments, prominences - magnetohydrodynamics (MHD) - waves - instabilities

\section{Introduction}

Solar prominences are magnetic suspensions of chromospheric plasma within the corona. This plasma is typically of the order of one hundred times cooler and denser than the surrounding atmosphere, and it accumulates in magnetic flux tubes which are anchored within the photosphere. From these flux tubes, a complex flux rope structure may emerge, which is highly sheared and with a twisted underlying magnetic structure. The twisted flux tubes are able to the capture the dense chromospheric plasma against gravity, and to thermally insulate it from the surrounding coronal plasma. This accumulation of plasma creates the solar prominence structures which we observe (Parenti 2014).

The formation of these magnetic structures and the mechanisms by which they are filled has long been studied. Historically a supporting topology of dipped field lines has been modelled by the weight of plasma suspension or by the dips in twisted flux ropes, as explained in reviews, for example, by Mackay et al. (2010). A recent review of the development of solar prominence models can be found in Gibson's 2018 review of models and theories (Gibson 2018), which demonstrates the history of the field well. Prominences are now often modelled as horizontallysheared flux ropes with arcade systems, arising as a result of dynamic solar surface motions and heating where highly polarised regions create polarity inversion lines above the photosphere, resulting in the formation of filament channels where these flux ropes can form.

As dynamic magnetic structures, a variety of formation processes have been proposed and modelled which can result in prominence structures. Examples of proposed formation processes include photospheric shearing motions (Zuccarello et al.
2015), and asymmetries in heating and structure which result in steady flows into asymmetrical loops (Klimchuk \& Luna 2019). They have even been modelled as dynamic threads not requiring loops, where thermal non-equilibrium mechanisms result in quasi-static structures (Karpen et al. 2001).

Mechanisms for transporting chromospheric material into the flux tubes broadly fall into the following three groups: levitation, injection, and evaporation (Priest 2014). In the former, a flux rope emerging from within the photospheric surface lifts plasma as the whole structure emerges into corona. In the case of injection, material is launched into the system by magnetic reconnection events, such as the local ejection of material from a site along a flux tube.

The final option, evaporation, causes an in situ accumulation of matter by localised heating above the footpoints of a flux tube leading to the evaporation of chromospheric material, which is carried along field lines. Above this heating, an accumulation of evaporated material causes a rise in density, which triggers radiative losses, and cooling. Condensation forms, which may be caught within the convex dips of twisted magnetic fields, suspending it against gravity. This explanation is considered the most likely method of transport and this method of mass accumulation has been well modelled in the literature (Gibson 2018).

Whichever mechanism explains the transport of the chromospheric plasma into the corona, its suspension against gravity relies on the topology of the underlying magnetic structures. Whilst a convex twist above the solar surface quickly drains new material back towards the footpoints where magnetic pressures eventually slow the descent, a prominence thread which is concave may hold the material in place similar to a bowl. This accumulation of material may lead to the stable growth of a 
prominence spine so long as the magnetic topology remains able to support it (Luna et al. 2012).

Observations of prominences show they are able to support oscillations across a range of scales (Engvold 2007) from the dynamic environment they inhabit. Examples of magnetohydrodynamic (MHD) waves travelling along the threads have been documented (Lin et al. 2007) since the development of tools with sufficient resolution to identify them. Torsional perturbations are found to be ubiquitous to these systems, with tornado-like features found along forming filaments ( $\mathrm{Su}$ et al. 2012), and within footpoints (Wedemeyer et al. 2013). They are suggested as potentially support prominence formation, though the exact nature of the motion is contested (for a discussion, see Luna et al. 2015).

The presence of Alfvén waves is also well supported since the launch of Hinode, which allows observations of the fine scale structure of prominence threads. Early results obtained with Hinode quickly showed oscillations along a prominence thread, caused by Alfvén waves (Okamoto et al. 2008). A recent study using the Swedish Solar Telescope was able to resolve high frequency torsional oscillations along a filament (Srivastava et al. 2017), with such torsional oscillations also observed to exist in filament barbs.

The present work aims to investigate the amplification of magnetic twists along a prominence thread by chromospheric evaporation: A stagnation point flow caused by chromospheric evaporation and the associated accumulation of plasma within a prominence thread, leading to the amplification of torsional perturbations. In the non-linear regime, the amplified twists may form the concave geometry required to support the uplifted plasma, and thus provide a mechanism for prominence formation. The twist amplification is due to a coupling mechanism by which the inflow acts as an energy source for the amplification of magnetic twists in a solar prominence.

A previous study, by Taroyan \& Soler (2019), showed the coupling of Alfvén waves with an inflowing plasma and an influx of torsional energy through a continuous driver at the footpoints could amplify small amplitude torsional perturbations. It was shown that this amplification was different from a resonance effect occurring in a static plasma. However, the question of whether the twist amplification would occur without the influx of torsional energy remained open.

The importance of the present work is the demonstration of twist amplification both with and without an influx of torsional wave energy through the footpoints. In the latter case, the amplification is solely the result of coupling between the stagnation point flow and the magnetic twists. Analytical solutions are constructed in terms of generalised hypergeometric functions which grow in time. We show that the inflow provides the energy required for the twist amplification. The results of the analytical study are confirmed numerically. The influx effects associated with the driven boundaries are eliminated. An initial pulse evolves along a segment of the thread where the inflow speed remains sub-Alfvénic.

The paper is constructed as follows: the model is constructed and the governing equations of the twists are presented in Sect. 2. The analytical and the numerical results are presented in Sects. 3 and 4. Our results are summarised and discussed in Sect. 5.

\section{Model}

We model a prominence thread as a single magnetic field line of length $L$, which plasma flows into through photospheric footpoints. The thread model is located between the photospheric boundaries at $-L / 2$ and $L / 2$, and it has a constant magnetic field strength $B$. Gravity and gas pressure play no role in the linear stage of the pulse evolution.

We aim to model the key features of solar prominence formation, in such a way that we can simulate a time-dependent plasma inflow along a thread which experiences an initial torsional perturbation. The analysis is carried out in the linear regime. It is well known that in a static medium, linear magnetic twists are decoupled from other motions. However, the time-dependent stagnation point flow introduces wave-flow coupling. We can perform the analysis without needing to currently address the back-reaction of the twists which should become important in the non-linear stage of the evolution.

\subsection{Inflow and density profiles}

Non-linear studies of plasma condensation in prominence threads (see, for example, Luna et al. 2012) predict the condensate to be captured first in the centre of the thread, and to accumulate so far as the thread topology allows. We model a basic, physically consistent prominence thread which assumes that density depends only on time, and not on position. Whilst our model is therefore not descriptively accurate, it represents a basic model of a prominence thread physically filling over time and it makes the problem of twist evolution mathematically tractable. This model allows us to then fix time-independent critical points within the thread where the flow speed becomes equal to the Alfvén speed, as shown in Fig. 1. Our model also lacks foot point driving which, along with the choice of a uniform density profile, is crucial for our investigation as we seek to investigate a system which limits energy influx. Previous work (Taroyan \& Soler 2019) has shown that a driven system may amplify twists over time. The uniform density profile and the initial value problem approach adopted in the present study eliminate the azimuthal energy influx through the boundaries, and fix time independent critical points within the thread where the flow changes from sub-Alfvénic to super-Alfvénic. The model constructed in this way allows us to demonstrate that the twist amplification process is fundamentally existent without any influx of azimuthal energy into the system. The prescribed density profile for our model is therefore:

$\rho=\rho_{0}\left(1+\frac{t}{t_{0}}\right)^{2}$,

where $\rho_{0}$ is the density at $t=0$, and $t_{0}$ is a constant.

A corresponding flow profile is obtained from the continuity equation:

$\frac{\partial \rho}{\partial t}+\frac{\partial}{\partial s}(\rho U)=0$

We substitute the density (1) into the continuity Eq. (2) and find the corresponding flow velocity:

$U=\frac{-2 s}{t+t_{0}}$.

The constant $t_{0}$ determines the initial flow speed. The flow profile has a stagnation point at $s=0$ and gradually vanishes as $t \rightarrow \infty$. It carries no azimuthal component, which will be important to note as we introduce azimuthal perturbations in our numerical simulations and intend to limit all azimuthal energy flux.

Whilst the choice of a spatially uniform density profile is a crude approximation of mass loading into a prominence, these 
velocity and density profiles allow for investigation of setups with a time independent Alfvén Mach number:

$M_{\mathrm{A}}=\frac{|U|}{C_{\mathrm{A}}}=\frac{2|s|}{C_{\mathrm{A} 0} t_{0}}$,

where $C_{\mathrm{A}}=B / \sqrt{\mu_{0} \rho}$ is the Alfvén speed and $C_{\mathrm{A} 0}=B / \sqrt{\mu_{0} \rho_{0}}$, the initial Alfvén speed. The Alfvén Mach number determines the locations of the critical points along the thread.

We utilise this time independent Alfvén Mach number to study the impact of both sub-Alfvénic and super-Alfvénic boundary inflows as well as exploring the behaviour around the fixed critical points where $M_{\mathrm{A}}=1$.

\subsection{Torsional perturbations}

To describe the evolution of perturbations along the thread we adopt a cylindrical coordinate system, with $\theta$ representing the azimuthal coordinate. The linear equations of motion and induction governing the azimuthal velocity, $v_{\theta}$, and magnetic field, $b_{\theta}$, are decoupled from the other MHD equations:

$\frac{\partial v_{\theta}}{\partial t}+U \frac{\partial v_{\theta}}{\partial s}=\frac{B}{\mu_{0} \rho} \frac{\partial b_{\theta}}{\partial s}$,

$\frac{\partial b_{\theta}}{\partial t}+\frac{\partial}{\partial s}\left(U b_{\theta}\right)=B \frac{\partial v_{\theta}}{\partial s}$,

where $U$ is the inflow velocity, $\rho$ is density, and $\mu_{0}$ is the magnetic permeability in vacuum. The evolution of linear twists is fully determined by the set of Eqs. (1), (3), (5) and (6). A gas pressure term that is consistent with the present profiles of density and flow velocity can be determined from the longitudinal component of the momentum equation which may also contain extra force terms. However, we may ignore this additional equation as long as the amplitudes of the perturbations remain small. The longitudinal component of the momentum equation should be included in the analysis during the non-linear stage of evolution, when the back reaction of the twists on the longitudinal flow through the ponderomotive force becomes important.

From these initial equations, the following wave energy equation has been previously derived by Taroyan \& Williams (2016):

$\frac{\partial W_{T}}{\partial t}+\frac{\partial F_{W}}{\partial s}=-\frac{\partial U}{\partial s} W_{m}$,

where $W_{T}$ is the sum of the kinetic and magnetic energy densities

$W_{T}=W_{k}+W_{m}=\frac{\rho v_{\theta}^{2}}{2}+\frac{b_{\theta}^{2}}{2 \mu_{0}}$

and $F_{W}$ represents the corresponding energy flux:

$F_{W}=U W_{T}-\frac{B}{\mu_{0}} v_{\theta} b_{\theta}$.

The source term on the right hand side of the energy Eq. (7) represents a coupling of twists and inflow along the thread, and the equation remains valid in the non-linear regime as shown by Williams \& Taroyan (2018).

The analysis of twist amplification cannot be performed using standard normal mode methods. In a normal mode analysis, linear perturbations are set proportional to $\exp (-\mathrm{i} \omega t)$ and the problem is reduced finding the eigenvalues $\omega$. Real values of $\omega$ correspond to stable oscillations, whereas complex values of $\omega$ with positive imaginary parts correspond to instability.

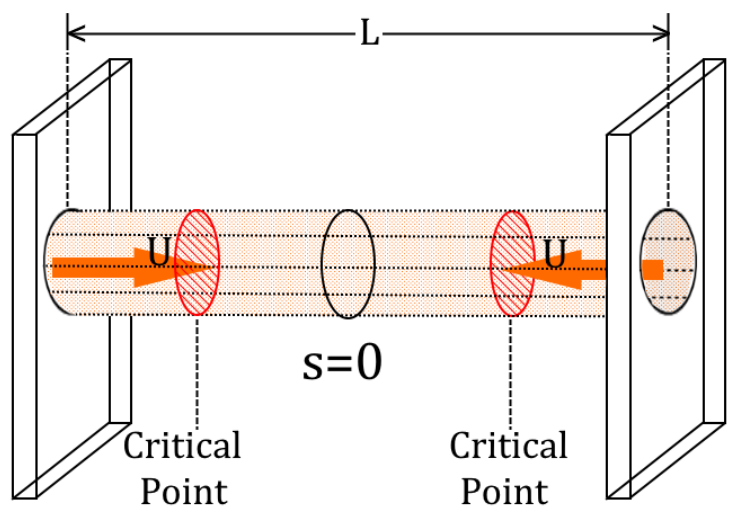

Photosphere Atmosphere Photosphere

Fig. 1. Prominence thread as a single magnetic field line of length $L$, with boundaries at $s= \pm \frac{1}{2} L$ anchored in a photosphere. A timedependent plasma inflow, $U$ along the thread is introduced, with a stagnation point at $s=0$ and a correspondingly uniform density profile. The critical points where the Alfvén speed equals the inflow speed are marked in red. We inject an initial torsional velocity pulse along the thread which propagates in opposite directions towards the two boundaries and follow the evolution of the pulse.

In the present case, the normal mode approach is not very useful due to the time dependent background. From a mathematical perspective, the coefficients of the governing Eqs. (5) and (6) depend both on time and distance. Therefore, the standard normal mode Fourier analysis is no longer applicable.

Another approach is to set a long condensation time-scale compared with the time-scale of the perturbations and tackle the problem in the WKB regime. For example, Erdelyi et al. (2011) studied the damping of longitudinal magneto-acoustic oscillations in slowly varying coronal plasma.

The time-scale of the perturbations is determined by the Alfvén speed, and the condensation time-scale is determined by the inflow speed. The ratio of the two speeds is not necessarily small in the present problem, which precludes the WKB approach. We adopt a different approach to make analytical progress.

\section{Analytical results}

The evolution of linear twists can be analysed by combining the equations of motion (5) and induction (6) into a single second order equation for $b_{\theta}$ :

$$
\begin{aligned}
\frac{\partial^{2} b_{\theta}}{\partial t^{2}}+\frac{\partial^{2}}{\partial t \partial s}\left(U b_{\theta}\right)+\frac{\partial}{\partial s}\left(U \frac{\partial b_{\theta}}{\partial t}\right)+ & \frac{\partial}{\partial s}\left(U \frac{\partial}{\partial s}\left(U b_{\theta}\right)\right) \\
& -C_{A}^{2} \frac{\partial^{2} b_{\theta}}{\partial s^{2}}=0 .
\end{aligned}
$$

Solutions to Eq. (10) are sought in the form:

$b_{\theta} \propto\left(t+t_{0}\right)^{\lambda} \hat{b}_{\theta}(s)$

where the unknown parameter $\lambda$ controls the rate of temporal growth or decline of $b_{\theta}$, and $\hat{b}_{\theta}$ is an unknown function of $s$. By substituting expression (11) and using the derived inflow profile (3) we eliminate the time variable $t$ from our governing Eq. (10):

$$
\begin{array}{r}
\lambda(\lambda-1) \hat{b}_{\theta}-2(\lambda-1) \frac{\mathrm{d}}{\mathrm{d} s}\left(s \hat{b}_{\theta}\right)-2 \lambda \frac{\mathrm{d}}{\mathrm{d} s}\left(s \hat{b}_{\theta}\right) \\
+4 \frac{\mathrm{d}}{\mathrm{d} s}\left(s \frac{\mathrm{d}}{\mathrm{d} s}\left(s \hat{b}_{\theta}\right)\right)-C_{\mathrm{A} 0}^{2} \frac{\mathrm{d}^{2} \hat{b}_{\theta}}{\mathrm{d} s^{2}}=0 .
\end{array}
$$


We introduce a new variable

$z=\frac{1}{2}\left(1+\frac{U}{C_{\mathrm{A}}}\right)=\frac{1}{2}\left(1-\frac{2 s}{C_{\mathrm{A} 0} t_{0}}\right)$

The change of variable (13) allows us to reduce Eq. (12) to the well-known hypergeometric equation:

$(1-z) z \hat{b}_{\theta}^{\prime \prime}+[c-(a+b+1) z] \hat{b}_{\theta}^{\prime}-a b \hat{b}_{\theta}=0$,

where the prime denotes differentiation with respect to $s$ and

$a=\frac{2-\lambda}{2}, \quad b=a+\frac{1}{2}, \quad c=\frac{1}{2}(a+b+1)$.

The hypergeometric differential equation has singular points at $z=0,1, \infty$ and, as a Fuchsian differential equation, it has linearly independent solutions of various forms in the neighbourhood of those points (Abramowitz \& Stegun 1964). We also note that $z=0$ and $z=1$ are the critical points where the Alfvén Mach number $M_{\mathrm{A}}=1$. Any perturbation generated within the sub-Alfvénic region $(0<z<1)$ will be travelling both in the negative direction at the local propagation speed $C_{\mathrm{A}}-U$ and in the positive direction at speed $C_{\mathrm{A}}+U$. In both cases, the propagation speed will vanish as the pulse approaches the critical points at $z=0$ and $z=1$, and it will be unable to travel further into the super-Alfvénic regions. We therefore require the solutions to vanish at the critical points $z=0$ and $z=1$.

We first focus on the solutions for $z=0$ that are represented in terms of the generalised hypergeometric functions ${ }_{2} F_{1}$ :

$\hat{b}_{\theta}=C_{12} F_{1}(a, b ; c ; z)+C_{2} z^{1-c}{ }_{2} F_{1}(a-c+1, b-c+1 ; 2-c ; z)$,

and which should vanish at the critical point $z=0$. Given that the hypergeometric function ${ }_{2} F_{1}$ may be represented by the power series:

${ }_{2} F_{1}(a, b ; c ; z)=1+\frac{a b}{c} z+\frac{a(a+1) b(b+1)}{c(c+1)} \frac{z^{2}}{2 !}+\cdots$,

we find that a non-trivial solution will vanish at $z=0$ only when $C_{1}=0$ and $1-c>0$. By substituting the expressions (15) into the second condition $1-c>0$ we derive the following inequality for $\lambda$ :

$1-\frac{1}{2} \times\left(\frac{2-\lambda}{2}+\frac{2-\lambda}{2}+\frac{1}{2}+1\right)>0$

which gives $\lambda>3 / 2$. In the neighbourhood of the critical point $z=1$ the solution is represented in the form:

$$
\begin{aligned}
\hat{b}_{\theta} & =C_{3}{ }_{2} F_{1}(a, b ; a+b+1-c ; 1-z) \\
& +C_{4}(1-z)^{c-a-b}{ }_{2} F_{1}(c-b, c-a ; c-a-b+1 ; 1-z) .
\end{aligned}
$$

It will vanish at $z=1$ only if $C_{3}=0$ and $c-a-b>0$. This second requirement again leads to the condition $\lambda>3 / 2$. Our solutions are therefore expressed in the form of Eq. (11), where $\lambda>3 / 2$ and $\hat{b}_{\theta}$ is expressed in terms of the generalised hypergeometric functions. Any superposition of these solutions with $\lambda>3 / 2$ will also represent a solution of the governing equation.

By this analysis we demonstrate that analytical solutions grow in time. This corresponds to an amplification of twisting. This is an important result as it demonstrates that the twists can amplify without the injection of azimuthal energy or azimuthal magnetic flux into the system: both $v_{\theta}$ and $b_{\theta}$ vanish at the critical points and beyond but growth occurs in the sub-Alfvénic region. The growth is caused by the wave-flow coupling along the thread that is represented by the source term in the right-hand side of Eq. (7).

\section{Numerical analysis of twist evolution}

The spatio-temporal evolution of the twists is further analysed numerically as an initial value problem. Distance is normalised with the respect to the length of the simulated thread, $L$, whilst speed is normalised with respect to the initial Alfvén speed, $C_{\mathrm{A} 0}=B / \sqrt{\mu_{0} \rho_{0}}$. Therefore, time is normalised with respect to the Alfvénic travel time $t_{\mathrm{A}}=L / C_{\mathrm{A} 0}$. The perturbations $v_{\theta}$ and $b_{\theta}$ are normalised with respect to $C_{\mathrm{A} 0}$ and $B$, correspondingly.

In accordance with the flow profile (3), the boundaries represent critical points when $t_{0}=1$ in normalised units. The value of $t_{0}$ therefore determines two distinct regimes within our work, corresponding to sub-Alfvénic and super-Alfvénic inflows at the boundaries. For this work we refer to these as sub-Alfvénic and super-Alfvénic regimes. For the sub-Alfvénic regime the flow is sub-Alfvénic at all points within the thread, whilst in the super-Alfénic regime there exist time-independent critical points within the thread where the flow becomes super-Alfvénic. This second regime might be applicable to active regions with higher inflow speeds. Our choice of the density profile (1) allows us to explore these regimes separately, due to the fixed position of the critical points along the thread.

The boundaries of the thread represent the photospheric footpoints at $s= \pm L / 2$ where we impose vanishing boundary conditions on the azimuthal velocities to ensure no influx of azimuthal kinetic energy. For sub-Alfvénic boundary flows, we note that equation (5) would then yield a second boundary condition, $\frac{\partial w_{\theta}}{\partial s}=0$, with $w_{\theta}=U \rho v_{\theta}-B b_{\theta} / \mu_{0}$ as a new variable (Taroyan \& Soler 2019). We note that this second condition is reduced to $\frac{\partial b_{\theta}}{\partial s}=0$ when $U$ is small, representing a Poynting flux used to buffer our magnetic field perturbations within the thread without further energy influx. We have applied this simpler condition to sub-Alfvénic flows with a maximum Mach number of $M_{\mathrm{A}}=0.2$ as tests produced negligible differences in outcomes. In summary, our boundary conditions for setups where the inflow profile is still sub-Alfvénic at the boundaries are:

$$
\begin{aligned}
& v_{\theta}=0, \\
& \frac{\partial b_{\theta}}{\partial s}=0 .
\end{aligned}
$$

For the super-Alfvénic regime, any pulse generated within the thread will always reach a critical point somewhere within the thread where the Alfvén speed is equal to the inflow speed and the pulse grinds to a halt. This allows us to use vanishing boundary conditions as we no longer need to be concerned with interactions at the boundaries, and so we are simply able to choose our second boundary condition as $b_{\theta}=0$. This eliminates all fluxes through the boundaries, and thus minimises the role of the boundaries. In the sub-Alfvénic regime, the vanishing boundary conditions for $v_{\theta}$ and $b_{\theta}$ are incompatible with the governing Eqs. (5) and (6) as additional conditions on the derivatives must be satisfied.

In the super-Alfvénic regime, one would expect to see no difference between the two boundary conditions. However, tests show differences in the evolution of magnetic twisting with vanishing boundaries as compared to using the conditions for a sub-Alfvénic boundary flow described above. In the latter case, we find numerical noise travelling beyond the critical points from the sub-Alfvénic region into the super-Alfvénic regions and disturbing the boundaries. The propagation of the disturbance against the super-Alfvénic flow is an indication of its numerical 
origin. In early runs with only 1000 spatial grid points this effect was observed as early as $t=7.0$, but we were able to suppress the effect until $t=18$ by increasing the spatial resolution to 5000 grid points, demonstrating this is a numeric effect. Further resolution increases were limited by excessive computation times. To minimise this effect and the resulting boundary interference we apply the vanishing boundary conditions:

$v_{\theta}=0$,

$b_{\theta}=0$.

We analyse the evolution of our model numerically by integrating a set of linear partial differential equations using the Advanced Maths and Statistics Library (IMSL_PDE_MOL) for IDL. The library uses the method of lines to solve for discrete spatial derivatives, with a continuous time variable. The work here uses a spatial grid made up of 5000 points.

At $t=0$ a torsional velocity pulse is applied to the system. We investigated various initial pulses in both the sub-Alfvénic and super-Alfvénic boundary flow regimes which would represent seed perturbations as an initial value problem. Physically we should expect perturbations in a dynamic atmosphere to be ubiquitous to the system, with them generated either local to thread, or pushed into the system but at all times present in some regard as it is unlikely that plasma in a dynamic solar atmosphere would carry no azimuthal disturbance. We here generate these as very small initial seed perturbations to avoid further energy influx through the boundaries, and to represent some initial perturbation profile as an initial condition.

Two initial setup categories were used in this study, both of which inject half-sine waves as:

$v_{\theta}(t, s)=A \cos (\pi s / l)$

where $A$ is the wave amplitude, $-l / 2<s<l / 2$, and $l$ is the pulse width. The setups either injected one single pulse, or randomly injected multiple pulses as described below.

The single pulse setups shown in this paper have amplitudes of $A=0.2$, for sub-Alfvénic inflows a width of $l=0.2$, and for a super-Alfvénic inflow a width of $l=0.6$. This pulse could either be centred about $s=0$ or offset.

For the random setup, rather than injecting a predefined number of pulses we define a probability for a pulse to be generated at a point. Every point along the s axis is then considered sequentially, such that pulses can be added one after the other, but are forbidden from overlapping. Starting from $s=-1 / 2$ we randomly determine if a new pulse should be injected, moving along the axis until a pulse is triggered. Once triggered, a random sign and amplitude are generated for a single pulse of width $l=0.1$ as defined above. No pulses are triggered for $s>0.4$ to ensure that the boundary conditions are maintained.

The generated pulses always split into two and travel in opposite directions along the thread, one towards each boundary. This means, for example, that a pulse generated with amplitude $A=0.2$ and width $l=0.2$ at $t=0$ will travel as two components along the thread, moving apart from each other with the same width but half the amplitude at $t=0.1$ (Fig. 2).

In testing, we used sine pulses of any width up to the length of the thread, with varying offsets, whilst the random wave setup was always made up of pseudo-randomly spaced sine waves of width $l=0.1$ across the length of the thread. The temporal and spatial evolution of a few of these pulse setups is presented in Figs. 2-4. (a)
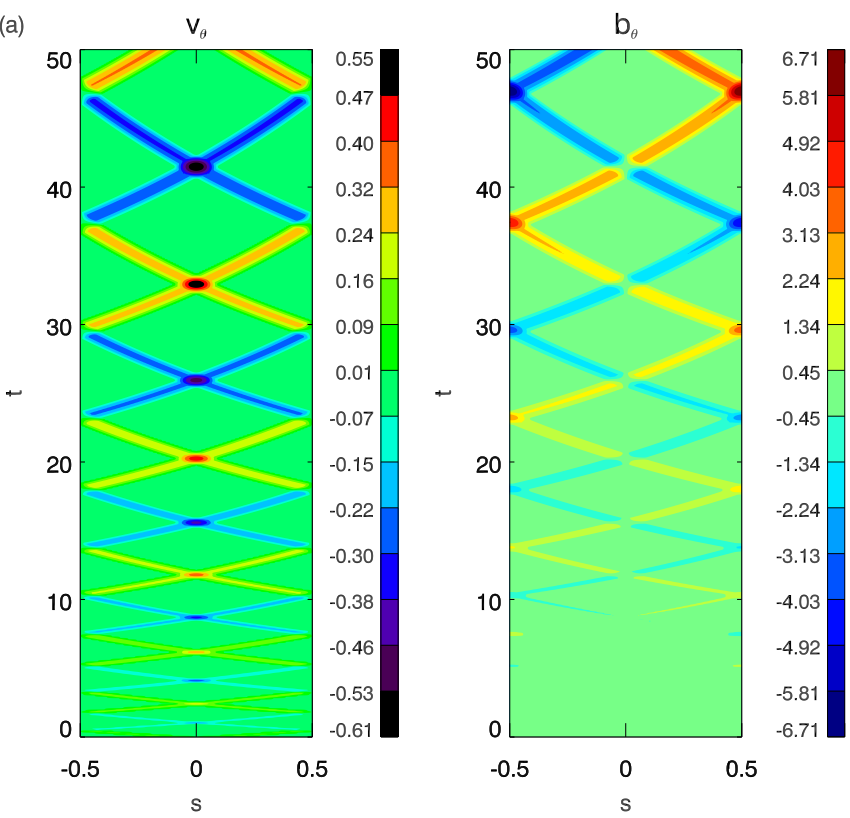

(b)

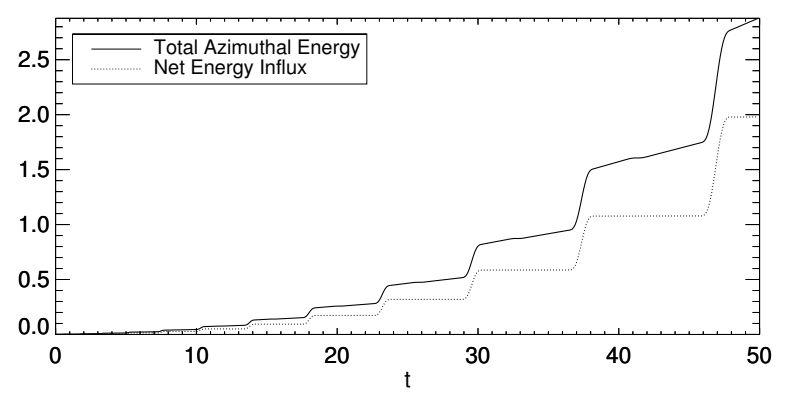

(c)

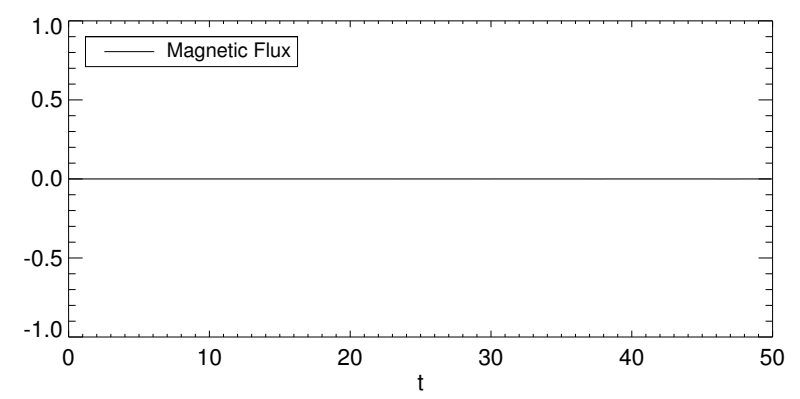

Fig. 2. (a) Contour plots showing the evolution of a single pulse centred at $s=0$ with a width of $l=0.2$. The selected parameter $t_{0}=5$ corresponds to an Alfvénic mach number of $M_{\mathrm{A}}=0.2$ at the boundaries. (b) The associated total azimuthal energy and the net energy influx through the boundaries. (c) The associated azimuthal magnetic flux, in this case at zero or negligible throughout.

\subsection{Case 1: Sub-Alfvénic flows}

To begin with we tested single pulse setups across the thread. In this, the simulations show a consistent pattern of growth in the magnetic field amplitude, which corresponds to an increase in the twisting of the magnetic field line.

Figures 2 and 3 show that the travel speed of the pulses slows as the simulation progresses. This is owing to an increase in the density, and a corresponding decrease in the Alfvénic and flow speeds.

An equation for the total azimuthal energy, $E=\int_{-L / 2}^{L / 2} W_{T} \mathrm{~d} s$, can be derived from Eq. (7): 
(a)

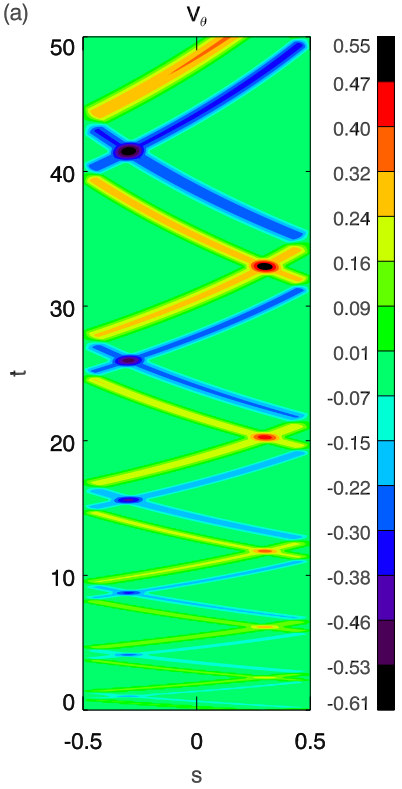

(b)

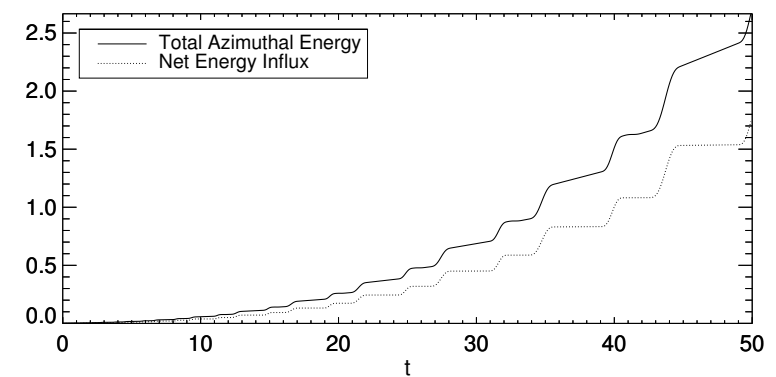

(c)

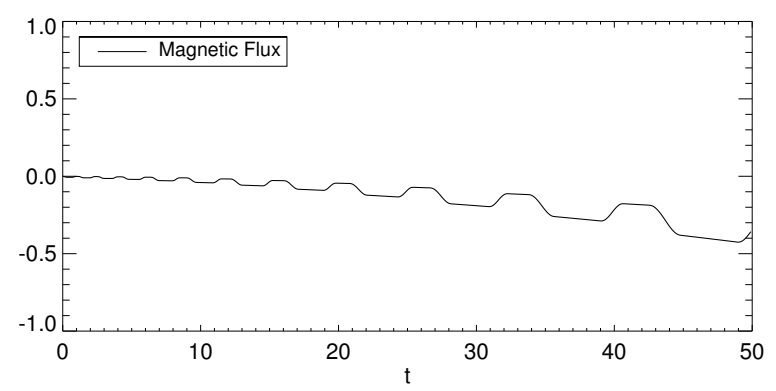

Fig. 3. (a) Contour plots showing the evolution of a single offset pulse with a $t_{0}=5$ and a width of $l=0.2$. (b) The associated total azimuthal energy and the net energy influx through the boundaries. (c) The associated magnetic flux, which increases in time due to the offset setup.

$$
\frac{\partial E}{\partial t}+\left.F_{W}\right|_{\frac{-L}{2}} ^{\frac{-L}{2}}=-\int_{-L / 2}^{L / 2} \frac{\partial U}{\partial s} W_{m} \mathrm{~d} s,
$$

such that if the second term in the left-hand side is zero then there is no influx or out-flux of energy, and no associated change in energy. The total azimuthal energies shown in the sub-Alfvénic Figs. 2-4 all grow, showing a distinct increase in total energy, separate from the influx, and with a step-like growth corresponding to wave-boundary interactions. This energy growth is a result of the source term on the right-hand side of Eq. (23) which represents the coupling of the decelerating flow with the twists along the thread. As we are conducting a linear analysis, we do not consider any back reactions on the background flow. However, in the non-linear stage of evolution the amplified twists are expected to affect the flow through the non-linear ponderomotive force. (a)
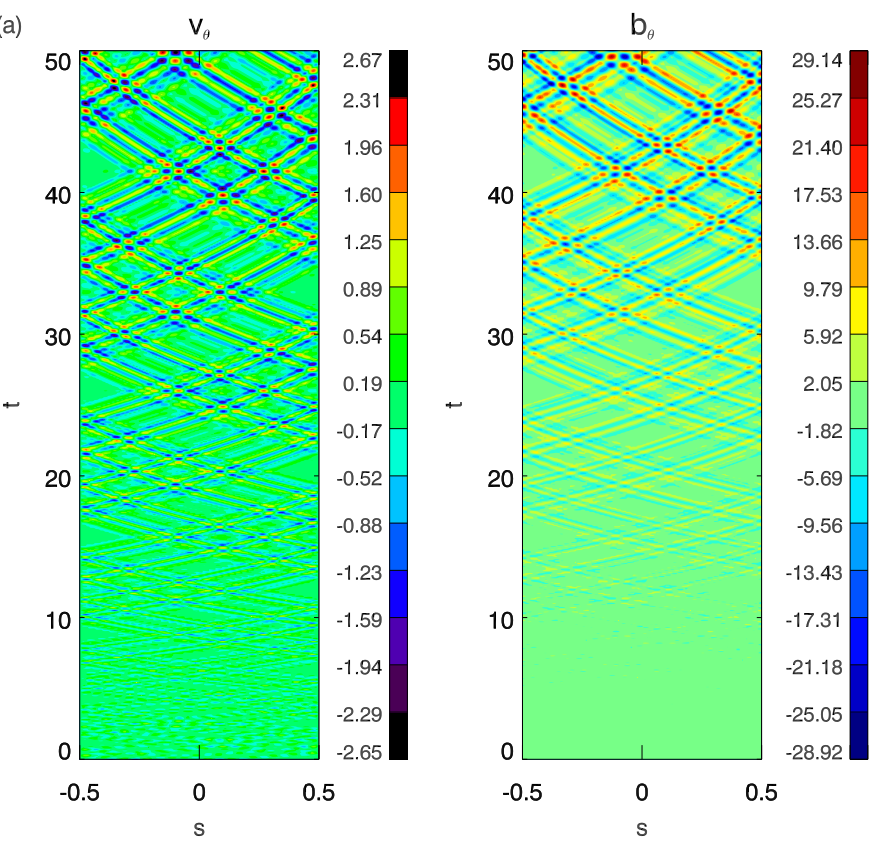

(b)

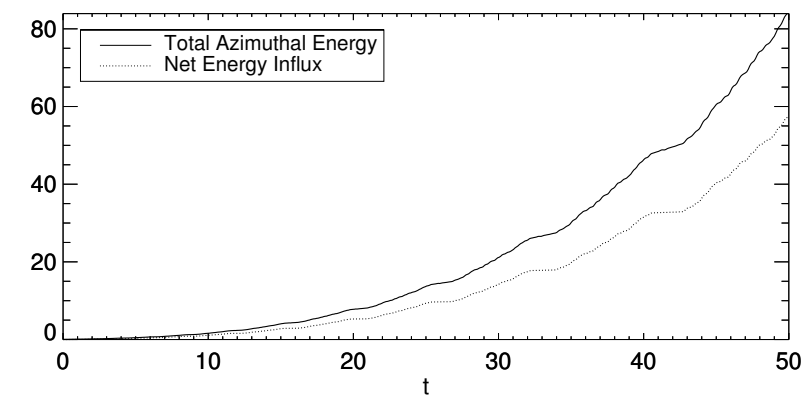

(c)

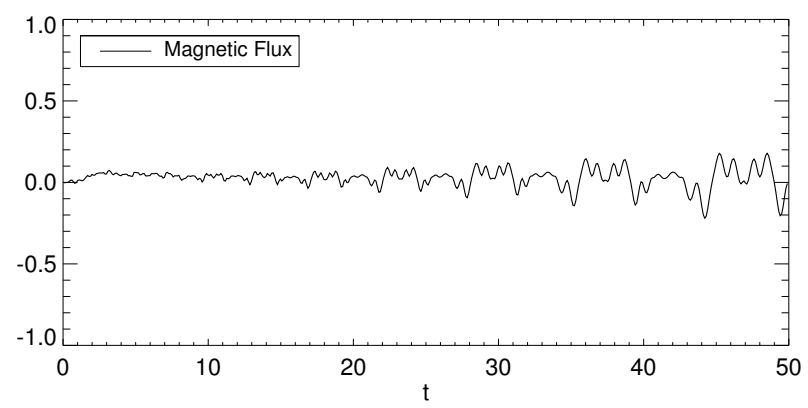

Fig. 4. (a) Contour plots showing the evolution of random pulses with $t_{0}=5$. (b) The associated total azimuthal energy and the net energy influx through the boundaries. (c) The associated magnetic flux, which remains low but increases over time.

This growth occurs as the pulse travels along the length of the thread, without any net energy influx, which is most easily seen in the energy graphs in Figs. 2 and 3. There is an additional step-like growth which occurs in total energy at the boundaries, where a net influx into the system at the boundaries causes a corresponding increase in total energy. This results in a step-pattern, with sharp total energy increases as the net energy increases at the boundaries and a smooth growth due to the coupling of the perturbation with the plasma inflow as the pulses travel across the thread. As the random setup in Fig. 4 has a much higher instance of interactions with the boundaries, the step increase is replaced with a smoother growth curve. However, there is still a distinctly separate increase in total energy aside from the influx. 
(a)
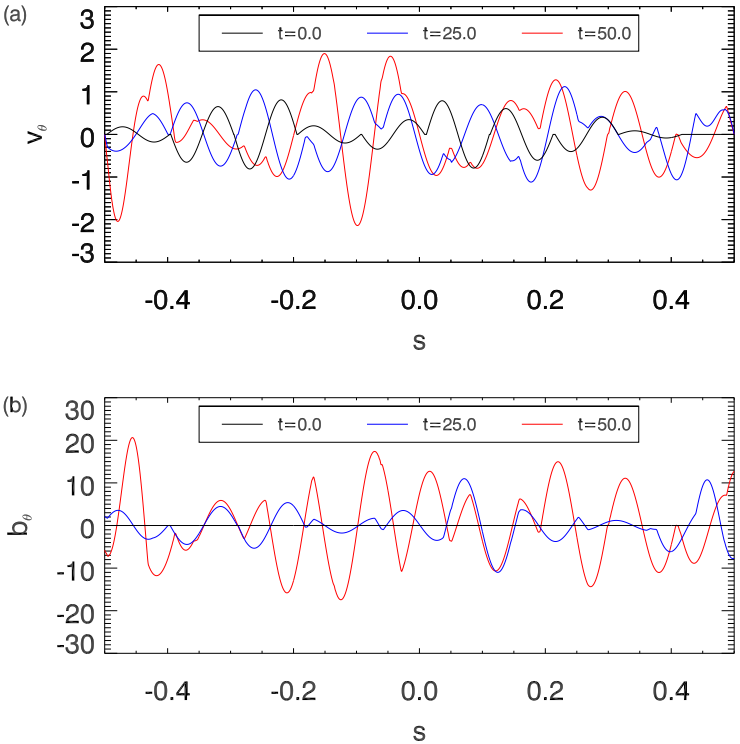

Fig. 5. (a) Profiles of $v_{\theta}$ at different moments of time for the random setup seen in Fig. 4. (b) Profiles of $b_{\theta}$ at different moments of time for the random setup seen in Fig. 4.

This increase can be attributed to the wave-flow coupling along the thread. The total energy is seen to amplify the rate of linear growth as the perturbations travel along the thread.

This pattern of growth as the perturbations travel along the thread is visible in all three setups, and is not a product of symmetry. This is demonstrated both in our use of offset initial conditions (as shown in Fig. 3), and by growth in random setups (as in Fig. 4). We also tested other values for the Alfvénic mach number and always observe twist amplification along the thread, with an increase in azimuthal energy separate from that caused by influx at the boundaries.

An equation for the total azimuthal magnetic flux can be derived from the induction Eq. (6):

$\frac{\partial}{\partial t} \int_{-L / 2}^{L / 2} b_{\theta} \mathrm{d} s+\left.U b_{\theta}\right|_{\frac{L}{2}} ^{\frac{L}{2}}=\left.B_{0} v_{\theta}\right|_{\frac{-L}{2}} ^{\frac{L}{2}}$.

The right-hand side of the above equation will always be 0 by our choice of boundary conditions. In the case of a symmetric initial pulse, the second term also becomes 0 . This explains why the magnetic flux remains unchanged in the third panel of Fig. 2. The symmetry is lost in the case of an offset pulse (Fig. 3) leading to gradual increase in the magnetic flux. However, there is no corresponding increase in the total energy.

Another graph representing the random setup used in Fig. 4 can be seen in Fig. 5, where the initial azimuthal perturbations and the evolution at different times is shown. The figure shows an increase in the azimuthal magnetic flux.

\subsection{Case 2: Super-Alfvénic flows}

When $t_{0}<1$ the critical points move into the thread domain. These critical points remain fixed in time and we can therefore set vanishing boundary conditions for both the velocity and the magnetic field, because in using this setup the perturbations will never reach the boundaries. Any pulse triggered along the thread quickly converges towards the critical points. In Fig. 6 we have used an initial pulse of width $l=0.6$ to capture the time where the perturbation was travelling along the thread. (a)

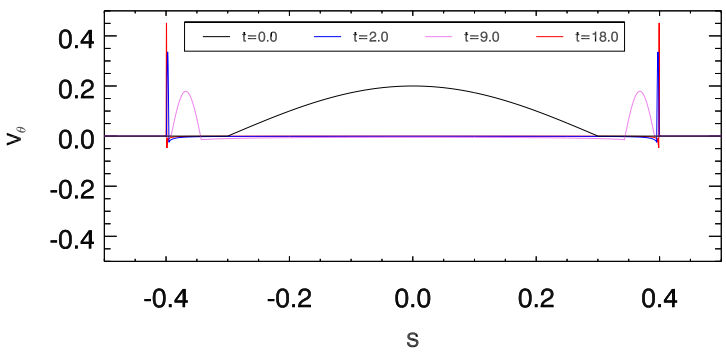

(b)

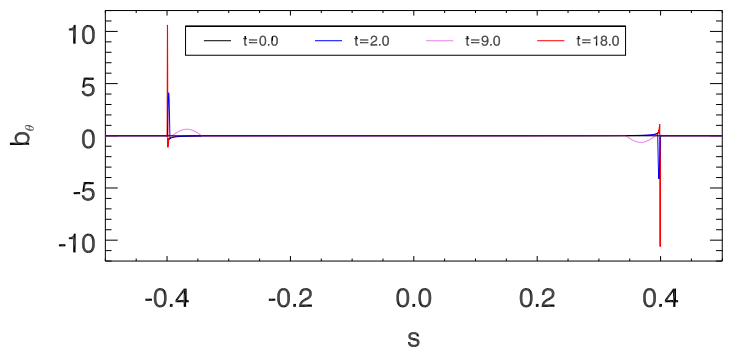

(c)

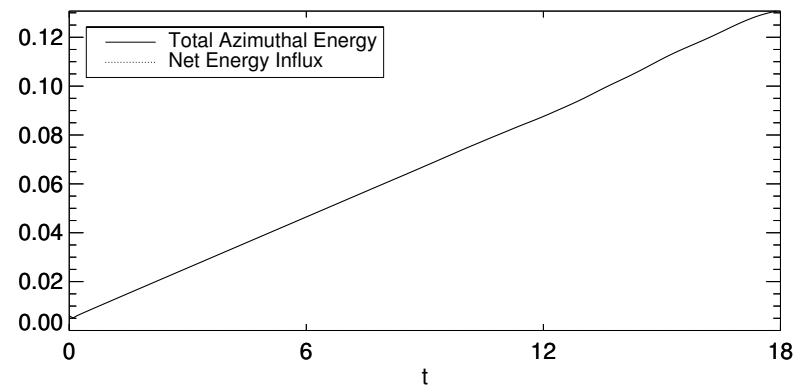

(d)

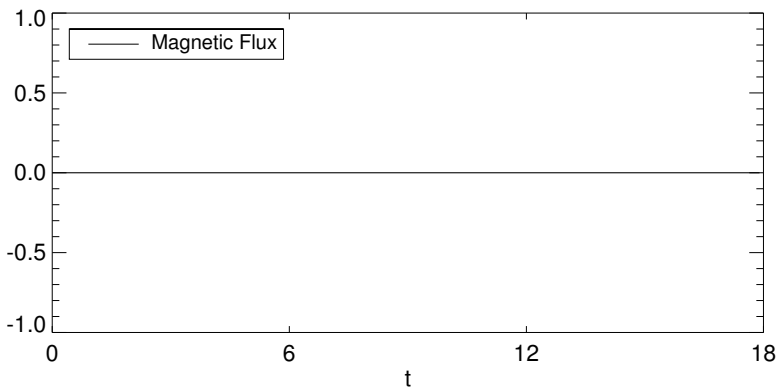

Fig. 6. (a) Evolution of $v_{\theta}$ for $t_{0}=0.8$ corresponding to a super-Alfvénic flow and critical points inside the thread. The initial pulse has a width of $l=0.6$ and the evolution of the pulse is shown up to $t=18$ when it approaches the critical points at about \pm 0.4 . (b) Evolution of $b_{\theta}$ for a 0.6 wide pulse up to $t=18$ showing the pulse propagating to the critical points at about \pm 0.4 . (c) The corresponding total energy of the system, up to $t=18$. (d) The total magnetic flux up to $t=18$ showing no variation until $t=18$.

At time $t=18$ we end the simulation as the pulse width becomes increasingly small as the pulse approaches the critical point where the inflow speed is equal to the Alfvén speed, reaching the limits of our spatial resolution. This steepening of an Alfvénic pulse at a critical point is a new result: The trailing edge of the pulse where the propagation speed is higher catches up with the leading edge where it is lower. The pulse eventually grinds to a halt as the propagation speed approaches 0 at the critical point. 
The steepening is accompanied by an increase in the total energy as can be seen in the third panel of Fig. 6 . The increase is solely due to the wave-flow coupling along the thread. There is no contradiction between the conservation of magnetic flux in panel $\mathrm{d}$ and the increasing total energy in panel $\mathrm{c}$, despite the increase in $b_{\theta}$; the total magnetic flux represented by the integral in Eq. 24 remains conserved due to the opposite signs at the critical points.

Generally, we find that these simulations show the same smooth increase along the thread as we find in the sub-Alfvénic flows, but without the net influx at the boundaries, until we reach the limits of our numerical resolution. Early runs of the superAlfvénic setups at lower spatial resolutions encountered numerical noise very quickly, but we were able to demonstrate the growth of total energy in the system was real by increasing the spatial resolution. A wider pulse choice also allows us to extend the time before the pulse steepens at the critical point. Even so, we limit the super-Alfvénic setup to $t=18$ both because of required higher spatial resolution, but also because we begin to generate very steep gradients in the magnetic field as the system evolves. The steep gradients mean that in the non-ideal induction equation:

$\frac{\partial b_{\theta}}{\partial t}+\frac{\partial}{\partial s}\left(U b_{\theta}\right)-\eta \frac{\partial^{2} b_{\theta}}{\mathrm{d} s^{2}}=B_{0} \frac{\partial v_{\theta}}{\partial s}$,

the diffusive term is no longer negligible and dissipation should be investigated. An investigation into this is planned as future work.

For the $t_{0}=0.8$ case shown in Fig. 6 we see a growth in total azimuthal energy. The growth continues after $t=18$, however, it is uncertain to what extent this growth is affected by numerical noise. This can be investigated further by including the diffusive term in the induction equation to determine how far this may suppress growth. Whilst the growth may be suppressed somewhat it will not be limited entirely, as this occurs only after the growth of steep gradients. This represents the limit at which our spatial resolution is insufficient and where our linearity assumptions will also begin to fail as $\partial B_{\theta}^{2}$ terms become important. It is unclear which of these effects will dominate first after the steepening occurs, but similar problems are addressed in the modelling of other non-linear processes, such as resonant absorption.

\section{Discussion and conclusions}

We have investigated the twisting of magnetic field lines driven by thread condensation. The density profile is dependent only on time, and not on position. The resulting flow speed and its gradient quickly vanish, in contrast to the profile used by Taroyan \& Soler (2019), and this has a negative impact on the process of twist amplification. Whilst the chosen uniform density profile is not a very accurate representation of the condensation process in prominence threads, it allows us to fix critical points that are time-independent. The pulse evolution can then be explored separately in the sub-Alfvénic and the super-Alfvénic regimes.

Another aim of the present study is the investigation of the pulse evolution in the absence of any azimuthal magnetic or energy flux into the system. We therefore try to minimise the role of the boundaries by considering an initial value problem. In the super-Alfvénic regime, the initial pulse does not reach the boundaries which eliminates the fluxes.

We first consider the problem analytically by imposing vanishing boundaries at the critical points. A physical justification for these conditions is the fact that the perturbations generated in the sub-Alfvénic region cannot reach the super-Alfvénic region as they will be swept back by the super-Alfvénic flow. These arguments are supported by the numerical results.

We construct analytical solutions by expressing them as a product of two functions. In the first we describe the spatial dependence in terms of generalised hypergeometric functions, whereas the temporal dependence is expressed in terms of a power law with a power law index of $\lambda>3 / 2$ indicating temporal growth.

The results of the analytical study are supported numerically. In the sub-Alfvénic regime, $b_{\theta}$ does not vanish and is subject to an amplification every time the pulse reaches the boundary. This results in a step-like amplification and energy increase that can be seen in Figs. 2 and 3. In between the steps, when there is no azimuthal influx, energy increases more gradually due to the coupling between the flow and the perturbations.

We find this growth in energy due to the coupling mechanism to be linear, but the rate of that linear growth is proportional to the flow derivative which, in the present model, vanishes proportionally to $1 /\left(t+t_{0}\right)$. On the other hand, the linear growth curve becomes steeper after each step as additional energy is pumped in.

The results from our study suggest that a plasma inflow along the thread leads to an amplification of twisting in the underlying magnetic field. This result is found regardless of the choice of initial perturbation, and crucially is seen in super-Alfvénic inflow simulations where there is no influx of azimuthal magnetic field or energy through the boundaries. In studies of wave propagation, waves of certain amplitude are usually required to be generated in the photosphere to have any significant effect on the atmosphere. The importance of the presented mechanism is the relaxation of this requirement. The amplification of the twists occurs through their coupling with the inflow.

In the super-Alfvénic setup, corresponding to the pulses converging at the critical points, the linear growth is not affected by the choice of boundary conditions. It is a feature that should be present in any decelerating plasma. That is to say that the coupling between the flow and linear Alfvénic perturbations is a fundamental phenomenon to plasma physics, and although we here focus on solar prominences this may have broader applications.

The spatio-temporal analysis of the pulse evolution in the super-Alfvénic regime clearly shows pulse steepening as it approaches the critical point. In approaching this point, the propagation speed is lower at the leading edge and higher at the trailing edge which leads to steepening of the pulse and the formation of small scales.

This formation of strong gradients combined with the linear increase in the total azimuthal energy is similar to the growth caused by a resonance. The process of resonant absorption is well known in plasma physics. It is the result of coupling between the incoming fast waves and the local Alfvén waves. The energy of the incoming wave is transferred into the local Alfvén waves which grow in amplitude.

However, in the present work, the coupling is between the decelerating flow and the propagating Alfvén waves which grind to a halt at the critical points. This novel mechanism requires further consideration with the inclusion of magnetic diffusivity and the non-linear effects. The resulting transfer of the wave energy into Joule heating and the non-linear effects caused by the steepening will be the subject of future study.

We also intend to construct a more realistic model by including the photospheric and chromospheric conditions, without the 
C. Sumner and Y. Taroyan: Magnetic Field Twist Amplification by Stagnation Point Flow

limitation in our choice of inflow and density profiles for timeindependent critical points. In such a model, a varying magnetic field and background plasma density will have conflicting influences; a stronger magnetic field will likely increase the flow speed and its gradient, whilst a higher plasma density is likely to lessen it.

Acknowledgements. Chloe Sumner would like to thank the STFC for their financial support (ST/S505225/1).

\section{References}

Abramowitz, M., \& Stegun, I. A. 1964, Handbook of Mathematical Function with Formulas, Graphs, and Mathematical Tables, ninth dover printing, tenth gpo printing edn. (New York: Dover)

Engvold, O. 2007, Proc. Int. Astron. Union, 3, 152

Erdelyi, R., Al-Ghafri, K. S., \& Morton, R. J. 2011, Sol. Phys., 272, 73

Gibson, S. 2018, Liv. Rev. Sol. Phys., 15,
Karpen, J. T., Antiochos, S. K., Hohensee, M., Klimchuk, J. A., \& MacNeice, P. J. 2001, ApJ, 553, L85

Klimchuk, J. A., \& Luna, M. 2019, ApJ, 884, 68

Lin, Y., Engvold, O., Rouppe van der Voort, L. H. M., \& van Noort, M. 2007, Sol. Phys., 246, 65

Luna, M., Karpen, J. T., \& DeVore, C. R. 2012, ApJ, 746, 30

Luna, M., Moreno-Insertis, F., \& Priest, E. 2015, ApJ, 808, L23

Mackay, D. H., Karpen, J. T., Ballester, J. L., Schmieder, B., \& Aulanier, G. 2010, Space Sci. Rev., 151, 333

Okamoto, T. J., Tsuneta, S., Berger, T. E., et al. 2008, Science, 318, 1577

Parenti, S. 2014, Liv. Rev. Sol. Phys., 11, 1

Priest, E. 2014, Magnetohydrodynamics of the Sun (New York: Cambridge University Press)

Srivastava, A. K., Shetye, J., Murawski, K., et al. 2017, Sci. Rep., 7

Su, Y., Wang, T., Veronig, A., Temmer, M., \& Gan, W. 2012, ApJ, 756, L41

Taroyan, Y., \& Williams, T. 2016, ApJ, 829, 107

Taroyan, Y., \& Soler, R. 2019, A\&A, 631, A144

Wedemeyer, S., Scullion, E., van der Voort, L. R., Bosnjak, A., \& Antolin, P. 2013, ApJ, 774, 123

Williams, T., \& Taroyan, Y. 2018, ApJ, 852, 77

Zuccarello, F. P., Aulanier, G., \& Gilchrist, S. A. 2015, ApJ, 814, 126 